\title{
Hara's theorem, quark model, and $\gamma_{5}$-dependent renormalization constants
}

\author{
P. Żenczykowski* \\ Dept. of Theor. Physics \\ Institute of Nuclear Physics \\ Radzikowskiego 152, 31-342 Kraków, Poland
}

April 23, 2017

\begin{abstract}
We discuss the applicability of $\gamma_{5}$-dependent field renormalization as a means of renormalizing away the apparent violation of Hara's theorem observed in the quark model. It is pointed out that a result totally analogous to the violation of Hara's theorem is predicted by the quark model also for neutral baryons. For neutral baryons, however, such a result cannot be renormalized away. This proves that $\gamma_{5}$-dependent renormalization does not provide a proper way for a hadron-level understanding of the violation of Hara's theorem observed in the quark model.
\end{abstract}

*E-mail:zenczyko@solaris.ifj.edu.pl 
Weak radiative hyperon decays (WRHD's) have been a challenge to our understanding for over 30 years. Despite all the work done during these years, a satisfactory theoretical understanding of these processes is still lacking. For a review of current theoretical and experimental situation in the field see ref.[1].

The puzzle posed by weak radiative hyperon decays centers on the issue of Hara's theorem [2] and its possible violation. Hara's theorem states that the parity-violating amplitude of decay $\Sigma^{+} \rightarrow p \gamma$ should vanish in the limit of $\mathrm{SU}(3)$ flavour symmetry. There are two results that seem to indicate that Hara's theorem is violated.

1. Since SU(3) symmetry is expected to be weakly broken one expects small parity-violating $\Sigma^{+} \rightarrow p \gamma$ amplitude (and, consequently, small decay asymmetry). Experiment [3] shows, however, that the asymmetry is large:

$$
\alpha\left(\Sigma^{+} \rightarrow p \gamma\right)=-0.72 \pm 0.086 \pm 0.045
$$

Furthermore, existing hadron-level Hara's-theorem-satisfying models lead to a pattern of asymmetries of three related WRHD's $\left(\Lambda \rightarrow n \gamma, \Xi^{0} \rightarrow \Lambda \gamma\right.$, $\Xi^{0} \rightarrow \Sigma^{0} \gamma$ ) that does not seem to be corroborated by experiment.

2. On the theoretical side it was observed by Kamal and Riazuddin [4] that Hara's theorem is violated in the quark model even in the case of exact SU(3)-symmetry. There have been several proposals of how to understand this quark model result [5, 6, 7]. All of them have various deficiencies. At present there is no consensus on how the result of Kamal and Riazuddin should be understood. The purpose of this note is to discuss the most recent proposal [8] in that matter.

In ref. [8] it has been argued that the apparent violation of Hara's theorem obtained in the quark model can be renormalized away at hadronic level by means of field renormalization with $\gamma_{5}$-dependent renormalization constants. Here we show that such a renormalization procedure, although applicable to fundamental charged fermions, cannot be successfully used for neutral baryons. 
Since quark model predicts violation of a theorem analogous to that of Hara also for neutral baryons, the conclusion is that the effect observed by Kamal and Riazuddin should not be associated with a possible need for a $\gamma_{5}$-dependent field renormalization. Thus, a proper way of understanding the quark model result must lie elsewhere.

Let us begin by writing down the most general form of (diagonal in flavour) vector and axial currents that couple to photon:

$$
\begin{gathered}
V_{\mu}=\bar{\psi}\left[f_{1}\left(q^{2}\right) \gamma_{\mu}+f_{2}\left(q^{2}\right) i \sigma_{\mu \nu} q^{\nu}+f_{3}\left(q^{2}\right) q_{\mu}\right] \psi \\
A_{\mu}=\bar{\psi}\left[g_{1}\left(q^{2}\right) \gamma_{\mu} \gamma_{5}+g_{2}\left(q^{2}\right) i \sigma_{\mu \nu} \gamma_{5} q^{\nu}+g_{3}\left(q^{2}\right) \gamma_{5} q_{\mu}\right] \psi
\end{gathered}
$$

As in ref. [8] the notation of [9] is used for Dirac matrices. CP-invariance requires reality of functions $f$ and $g$ if standard form of Dirac equation is used. (In ref. [B] formfactors $f_{3}$ and $g_{2}$ are considered imaginary which is incompatible with the requirement of CP-invariance (see ref.[10]).)

There are two types of $\gamma_{5}$-dependent transformations of Dirac spinors:

1) "phase" transformation

$$
\psi^{\prime}=\exp \left(i \alpha \gamma_{5}\right) \psi
$$

2) "scale" transformation

$$
\psi^{\prime}=\exp \left(\beta \gamma_{5}\right) \psi
$$

with real $\alpha, \beta$. The adjoint spinors transform like $\overline{\psi^{\prime}}=\bar{\psi} \exp \left(i \alpha \gamma_{5}\right)$ and $\overline{\psi^{\prime}}=\bar{\psi} \exp \left(-\beta \gamma_{5}\right)$ respectively. Spinors $\psi_{L}$ and $\psi_{R}$ transform under phase transformations with opposite phases while under scale transformations their relative size is changed.

Let us discuss how the form of currents $V_{\mu}, A_{\mu}$ is affected by phase and scale transformations. Using properties of $\gamma$ matrices we find

$$
\exp \left(-i \alpha \gamma_{5}\right)\left[\gamma_{\mu}, \gamma_{\mu} \gamma_{5}\right] \exp \left(-i \alpha \gamma_{5}\right)=\left[\gamma_{\mu}, \gamma_{\mu} \gamma_{5}\right]
$$




$$
\begin{aligned}
\exp \left(-i \alpha \gamma_{5}\right)\left[\sigma_{\mu \nu}, \sigma_{\mu \nu} \gamma_{5}, \gamma_{5}\right] \exp \left(-i \alpha \gamma_{5}\right) & =\left[\sigma_{\mu \nu}, \sigma_{\mu \nu} \gamma_{5}, \gamma_{5}\right]\left(c-i s \gamma_{5}\right) \\
\exp \left(\beta \gamma_{5}\right)\left[\sigma_{\mu \nu}, \sigma_{\mu \nu} \gamma_{5}, \gamma_{5}\right] \exp \left(-\beta \gamma_{5}\right) & =\left[\sigma_{\mu \nu}, \sigma_{\mu \nu} \gamma_{5}, \gamma_{5}\right] \\
\exp \left(\beta \gamma_{5}\right)\left[\gamma_{\mu}, \gamma_{\mu} \gamma_{5}\right] \exp \left(-\beta \gamma_{5}\right) & =\left[\gamma_{\mu}, \gamma_{\mu} \gamma_{5}\right]\left(c_{h}-s_{h} \gamma_{5}\right)
\end{aligned}
$$

where $c=\cos 2 \alpha, s=\sin 2 \alpha, c_{h}=\cosh 2 \beta, s_{h}=\sinh 2 \beta$.

Under phase transformations the standard form of currents (Eqs.(2, 3)) transforms therefore to

$$
\begin{gathered}
V_{\mu}^{\prime}=\overline{\psi^{\prime}}\left[f_{1} \gamma_{\mu}+\left(c f_{2}-i s g_{2}\right) i \sigma_{\mu \nu} q^{\nu}+\left(c f_{3}-i s g_{3}\right) q_{\mu}\right] \psi^{\prime} \\
A_{\mu}^{\prime}=\overline{\psi^{\prime}}\left[g_{1} \gamma_{\mu} \gamma_{5}+\left(c g_{2}-i s f_{2}\right) i \sigma_{\mu \nu} \gamma_{5} q^{\nu}+\left(c g_{3}-i s f_{3}\right) \gamma_{5} q_{\mu}\right] \psi^{\prime}
\end{gathered}
$$

From Eqs.(7.8) we see that: 1) functions $f_{1}^{\prime}\left(=f_{1}\right), g_{1}^{\prime}\left(=g_{1}\right)$ are unaffected by phase transformations and 2) functions $f_{2}^{\prime}\left(=c f_{2}-i s g_{2}\right), f_{3}^{\prime}, g_{2}^{\prime}, g_{3}^{\prime}$ may in general be complex even though $\mathrm{CP}$ is conserved. However, the form of the Dirac equation has to be modified then to (compare ref.[1])

$$
\left(\not p-m\left(c-i s \gamma_{5}\right)\right) \psi^{\prime}=0
$$

Similarly, under scale transformations the standard form (Eq.(2, 3) ) of currents $V_{\mu}, A_{\mu}$ transforms to

$$
\begin{gathered}
V_{\mu}^{\prime}=\overline{\psi^{\prime}}\left[f_{1}^{\prime} \gamma_{\mu}+f_{2} i \sigma_{\mu \nu} q^{\nu}+f_{3} q_{\mu}\right] \psi^{\prime} \\
A_{\mu}^{\prime}=\overline{\psi^{\prime}}\left[g_{1}^{\prime} \gamma_{\mu} \gamma_{5}+g_{2} i \sigma_{\mu \nu} \gamma_{5} q^{\nu}+g_{3} \gamma_{5} q_{\mu}\right] \psi^{\prime}
\end{gathered}
$$

where $f_{1}^{\prime}=c_{h} f_{1}-s_{h} g_{1}, g_{1}^{\prime}=c_{h} g_{1}-s_{h} f_{1}$, and $f_{2}=f_{2}^{\prime}$ etc. i.e. only the coefficients at the $\gamma_{\mu}, \gamma_{\mu} \gamma_{5}$ terms are modified. Hereafter the prime sign $\left({ }^{\prime}\right)$ is used to label functions $f, g$ when they correspond to a non-standard form of Dirac equation.

We are now prepared to discuss the applicability of $\gamma_{5}$-dependent renormalization to neutral baryons. We follow the argument of ref.[B] closely. For simplicity consider just a neutron. Near its mass-shell and in the absence of weak interactions (but with complete account for strong and electromagnetic interactions) the neutron propagator has the form

$$
S_{o}^{-1}=\not p-m_{o}
$$


Let us now set the Cabibbo angle to zero. With weak interactions turned on the propagator of Eq.(12) is modified and close to its new mass-shell it has the general form (see ref.[[]])

$$
S^{\prime-1}=a \not p+b \not p \gamma_{5}-m^{\prime}
$$

In writing Eq.(13) we have assumed that an appropriate phase transformation has been already carried out to bring the general mass term of the form given in Eq.(9) to the standard form. Such a transformation does not affect the $a \not p$ and $b \not p \gamma_{5}$ terms (compare Eq.(6) ). Since weak interactions are small perturbations we have $a \approx 1,|b / a| \ll 1$, and $m^{\prime} \approx m_{o}$. Let us now bring the propagator of Eq.(13) to the standard Dirac form. In order to achieve this we perform a scale renormalization with appropriate parameter $\beta$ :

$$
\begin{aligned}
S^{-1} & =\exp \left(-\beta \gamma_{5}\right) S^{\prime-1} \exp \left(+\beta \gamma_{5}\right) \\
& =\left(a c_{h}+b s_{h}\right) \not p^{\prime}+\left(b c_{h}+a s_{h}\right) \not \gamma_{5}-m^{\prime}
\end{aligned}
$$

Thus, in order to bring neutron propagator to its standard Dirac form we need $\tanh (2 \beta)=-b / a$, i.e. $\beta$ of order $b / a$.

Renormalization of propagators as in Eq.(14) is associated with a simultaneous renormalization of fields $\left(\psi^{\prime} \rightarrow \psi\right.$, as in Eq.(5) $)$ and of the form of currents (Eqs.(10,11)). Since we require that, after renormalization, neutron couplings to photon satisfy $f_{1}(0)=0$ (zero charge) and $g_{1}(0)=0$ (analog of the assumption necessary for the proof of Hara's theorem) we obtain the conditions

$$
\begin{aligned}
& f_{1}(0)=f_{1}^{\prime}(0) c_{h}+g_{1}^{\prime}(0) s_{h}=0 \\
& g_{1}(0)=g_{1}^{\prime}(0) c_{h}+f_{1}^{\prime}(0) s_{h}=0
\end{aligned}
$$

Assume now that in perturbative calculations in some model we have obtained a nonvanishing $g_{1}^{\prime}(0)$. Whatever value of $f_{1}^{\prime}(0)$ is obtained it is clear that renormalization conditions of Eqs.(15) require $f_{1}^{\prime}(0) / g_{1}^{\prime}(0)=-\tanh (2 \beta)=$ $-1 / \tanh (2 \beta)$. Thus, in particular, $\beta= \pm \infty$ is required. This cannot be reconciled with the perturbative renormalization condition that $\beta$ is to be of order $|b / a| \ll 1$. Thus, if a nonzero $g_{1}^{\prime}(0)$ is somehow generated for neutral baryons, it cannot be renormalized away by a $\gamma_{5}$-dependent transformation. 
Let us now show that in the quark model the perturbative calculation of the contribution from $W$-exchange between quarks does indeed lead to $g_{1}^{\prime}(0) \neq 0$ for neutron $n$. To see this observe that neutron spin-flavour wave function (quarks: $d d u$ ) is obtained from that of proton (quarks: $u u d$ ) by a simple replacement $u \leftrightarrow d$. Symmetry of the wave function ensures that it is sufficient to consider $W$-exchange in one $u d$ diquark only. For the proton the photon-proton parityviolating coupling can be expressed in terms of photon-diquark couplings as

$$
\langle p \uparrow \gamma|T| p \downarrow\rangle=\frac{1}{3 \sqrt{2}} t_{+1}-\frac{1}{3 \sqrt{2}} t_{-1}-\frac{1}{\sqrt{2}} v
$$

where parity violating weak+electromagnetic diquark $\rightarrow$ diquark $+\gamma$ transition amplitudes are defined as

$$
\begin{aligned}
t_{+1} & =\left\langle S^{+1}(u d) \gamma|T| A(u d)\right\rangle \\
t_{-1} & =\left\langle A(u d) \gamma|T| S^{-1}(u d)\right\rangle \\
v & =\left\langle S^{0}(u d) \gamma|T| S^{-1}(u d)\right\rangle+\left\langle S^{+1}(u d) \gamma|T| S^{0}(u d)\right\rangle
\end{aligned}
$$

with diquark states

$$
\begin{aligned}
|A(u d)\rangle & =\left|\frac{1}{\sqrt{2}}(u d-d u) \frac{1}{\sqrt{2}}(\uparrow \downarrow-\downarrow \uparrow)\right\rangle \\
\left|S^{+1}(u d)\right\rangle & =\left|\frac{1}{\sqrt{2}}(u d+d u) \uparrow \uparrow\right\rangle \\
\left|S^{0}(u d)\right\rangle & =\left|\frac{1}{\sqrt{2}}(u d+d u) \frac{1}{\sqrt{2}}(\uparrow \downarrow+\downarrow \uparrow)\right\rangle \\
\left|S^{-1}(u d)\right\rangle & =\mid \frac{1}{\sqrt{2}}(u d+d u) \downarrow \downarrow
\end{aligned}
$$

For the neutron we get similarly

$$
\langle n \uparrow \gamma|T| n \downarrow\rangle=-\frac{1}{3 \sqrt{2}} t_{+1}+\frac{1}{3 \sqrt{2}} t_{-1}-\frac{1}{\sqrt{2}} v
$$

Calculations in the quark model as in ref. [⿴囗十 give $t_{+1}=-t_{-1}$ and $v=0$ and, consequently, they yield equal (up to a sign) nonzero values of $g_{1}^{\prime}(0)$ for proton and neutron. The origin of the nonzero value of $g_{1}^{\prime}(0)$ (whatever it is) is clearly the same for both proton and neutron.

The above argument may be dressed in a slightly more elaborate form by following the lines of ref. [8]. Namely, one can consider a Cabibbo-suppressed 
charm-changing weak radiative transition $\Sigma_{c}^{0} \rightarrow n \gamma(c d d \rightarrow u d d+\gamma)$. For this process an analog of Hara's theorem is expected to hold: in the limit of equal masses of the $u$ and $c$ quarks the parity violating $\Sigma_{c}^{0} \rightarrow n \gamma$ amplitude should vanish. The reasoning of ref. [8] may then be applied. First, we imagine a world in which $u$ and $c$ quarks are degenerate. By forming appropriate linear combinations of $u$ and $c$ quarks $\left(u^{\prime}, c^{\prime}\right)$ one can then eliminate the $c^{\prime} \rightarrow d$ transition in the Cabibbo matrix and deal with weak $W$-exchange-induced $u^{\prime} d \rightarrow d u^{\prime}$ transition exclusively (and a vanishing $c^{\prime} d \rightarrow d c^{\prime}$ transition). States $n^{\prime}$ and $\Sigma_{c}^{\prime o}$ are then not transformed into each other by a single $W$-exchange process. In this way we diagonalize the problem and are led to consider process $u^{\prime} d d \rightarrow u^{\prime} d d+\gamma$ i.e. an $n^{\prime} \rightarrow n^{\prime} \gamma$ coupling. This is essentially what we were discussing previously with an unimportant change of names $\left(n^{\prime} \rightarrow n\right)$.

Inability of the $\gamma_{5}$-dependent field renormalization to explain the origin of quark-model violation of Hara's theorem is clearly related to the composite nature of baryons as prescribed by the quark model. Indeed, the $W$-exchange contribution which lies at the origin of quark-model violation of Hara's theorem "does not know" about the charge of the spectator quark. Thus the total baryon charge may be reduced to zero by assuming an appropriate charge on the spectator quark. Discussion of this paper shows therefore that if the result of Kamal and Riazuddin is to be understood at some composite level it should probably be the diquark level.

In summary we have shown that: 1) quark model does lead to a nonvanishing $g_{1}^{\prime}(0)$ for neutron-photon couplings and 2) this contribution cannot be renormalized away by a $\gamma_{5}$-dependent transformation.

Consequently, $\gamma_{5}$-dependent renormalization does not provide a proper way for a hadron-level understanding of the violation of Hara's theorem in the quark model.

\section{ACKNOWLEDGEMENTS.}

This work was partially supported by the KBN grant No 2P03B23108. 


\section{References}

[1] J. Lach and P. Żenczykowski, Int. J. Mod. Phys. A10, 3817 (1995).

[2] Y. Hara, Phys. Rev. Lett. 12, 378 (1964).

[3] M. Foucher et al., Phys. Rev. Lett. 68, 3004 (1992).

[4] A. N. Kamal and Riazuddin, Phys.Rev. D28, 2317 (1983).

[5] L.-F.Li and Y.Liu, Phys. Lett. B195, 281 (1987). See also M. K. Gaillard, Phys. Lett. B211, 189 (1988).

[6] P. Żenczykowski, Phys. Rev. D40, 2290 (1989); ibid D44, 1485 (1991).

[7] V. Dmitrašinović, Phys. Rev. D54, 5899 (1996). See also P. Żenczykowski, "Comment on 'Hara's theorem in the constituent quark model", preprint INP 1765/PH, hep-ph/9709230.

[8] Ya. Azimov, Z. Phys. A359, 75 (1997).

[9] J. D. Bjorken, S. D. Drell, Relativistic Quantum Mechanics (McGraw-Hill, N.Y., 1964).

[10] L. B. Okun, Weak Interactions of Elementary Particles (Pergamon, 1965), Chapter 5 .

[11] J. F. Donoghue, E. Golowich, and B. R. Holstein, Dynamics of the Standard Model (Cambridge University Press, 1992), Chapter II-1. 